\title{
Incidence of $\beta$-lactamase producing Escherichia coli amongst clinical isolates in Dhaka, Bangladesh
}

\author{
Anindita Bhowmik ${ }^{1}$, Sunjukta Ahsan ${ }^{2 *}$, Marufa Zerin Akhtar ${ }^{3}$ and Md. Shahidul Kabir ${ }^{4}$ \\ ${ }^{I}$ Department of Microbiology, Jagannath University, Bangladesh; ${ }^{2}$ Department of Microbiology, University of \\ Dhaka, Dhaka 1000, Bangladesh; ${ }^{3}$ Department of Microbiology, University of Dhaka, Dhaka 1000, \\ Bangladesh; ${ }^{4}$ Department of Microbiology, Stamford University Bangladesh, 51 Siddeshwari Road, Dhaka- \\ 1217, Bangladesh
}

Received 23 April 2015/Accepted 09 June 2015

\begin{abstract}
Escherichia coli is one of the most important clinical pathogens that demonstrate antibiotic resistance through their $\beta$-lactamase activity. The present study reflected on the presence of $\beta$-lactam antibiotic resistance in clinical samples. Antibiotic resistance patterns of 20 clinical isolates of Escherichia coli against $\beta$-lactam antibiotics were investigated. These isolates were collected from a hospital in Dhaka city and MIC values were determined against Imipenem, Cefotaxime and Ceftriaxone. In case of Ceftriaxone the highest MIC was $>4096 \mu \mathrm{g} / \mathrm{mL}$ for $50 \%$ isolates with a minimum MIC of $1 \mu \mathrm{g} / \mathrm{ml}$ for $10 \%$ isolates. In case of Cefotaxime, MIC values ranged between $1 \mu \mathrm{g} / \mathrm{ml}$ and $>4096 \mu \mathrm{g} / \mathrm{ml}$ for $20 \%$ and $10 \%$ isolates, respectively. In contrast, $80 \%$ of the isolates had an MIC of Imipenem at $1 \mu \mathrm{g} / \mathrm{mL}$ with only $5 \%$ isolate having MIC of $8 \mu \mathrm{g} / \mathrm{mL}$. Eight out of $20(40 \%)$ isolates were ESBL producers, 14 (70\%) were $\beta$-lactamase producers. Two isolates exhibited ESBL activity against Cefotaxime and Ceftriaxone, 3 against only Cefotaxime and 3 against only Ceftriaxone. Ceftriaxone resistance in 10 randomly picked isolates was found to be stable for 21days in stability assay. Considering the findings of the present study, it appears that Imipenem still remains the drug of choice for $E$. coli infections; alternatively, combination therapy may be prescribed. In vitro stability of Ceftriaxone resistance raises concern from a public health point of view as this may aid antibiotic resistance transfer to other bacteria in the environment.
\end{abstract}

Key words: Antibiotic resistance; E. coli; Minimum Inhibitory Concentration (MIC); $\beta$-lactam antibiotics; Extended Spectrum Beta Lactamase (ESBL) producers; Stability assays

Antibiotic resistance occurs when bacteria change to protect themselves from an antibiotic. They are then no longer sensitive to that antibiotic. When this happens, antibiotics that previously would have killed the bacteria or stopped them from multiplying, will no longer work. Antimicrobial resistance (AMR) threatens the effective prevention and treatment of an everincreasing range of infections caused by bacteria, parasites, viruses and fungi. It is an increasingly serious threat to global public health that requires action across all government sectors and society. Bacteria can become resistant to antibiotics by mutating (changing) their genes after being in contact with an antibiotic. These changes allow the bacteria to survive or 'resist' the antibiotic. Unfortunately, bacteria can also develop antibiotic resistance through contact with other bacteria. Resistant bacteria can pass their genes to other bacteria, forming a new antibiotic resistant 'strain' of the bacteria. Resistant strains of bacteria can spread to other people though faecal oralroute or anv other vectors.

"Corresponding Author: Mialing addess. Dr. Sunjukta Ahsan, Department of Microbiology, University of Dhaka, Dhaka 1000, Bangladesh. Email: Sunjukta@du.ac.bd.
Mechanisms of antibiotic resistance in bacteria are varied and also include target protection, target substitution, antibiotic detoxification and block of intracellular antibiotic accumulation. Resistance may also be mediated by plasmid, mobile genetic elements such as integron or transposon, mutation in chromosomal DNA, $\beta$ lactamase or efflux pump. Acquisition of genes needed to elaborate the various mechanisms is greatly aided by a variety of promiscuous gene transfer systems, such as bacterial conjugative plasmids, transposable elements and integron systems that move genes from one DNA system to another and from one bacterial cell to another, not necessarily one related to the gene donor (1). Specific growth states (e.g. biofilm formation and anaerobiosis) can also negatively impact antimicrobial susceptibility (2). In recent years, it has been shown that a substantial portion of the resistance genes present on the plasmids and transposons of gramnegative bacilli are integrated into DNA elements called "integrons" (3). The dissemination of members of the Enterobacteriacae family with extended spectrum betalactamases (ESBLs) and metallo-beta-lactamases (MBLs) has become a global concern. ESBLs and MBLs have been reported in humans, domestic animals, wildlife, the 
environment and their isolation frequencies are increasing rapidly worldwide. Most studies have been performed in developed countries and quite a few in developing countries, where the antibiotic consumption is often poorly controlled (4). This study was conducted to identify patterns of $\beta$-lactam antibiotic resistance in selected clinical $E$. coli isolates, to investigate production of $\beta$-lactamase by the test bacteria, to determine the Minimum Inhibitory Concentration (MIC) of the test bacteria to selected $\beta$-lactam antibiotics viz. Ceftriaxone, Cefotaxime and Imipenem to categorize the test isolates as ESBL or non-ESBL producers and to demonstrate the stability of ceftriaxone resistance in selected test isolates.

\section{MATERIALS AND METHODS}

Sample history. A total of twenty clinical samples were collected from a renowned hospital of Dhaka city. Samples were maintained in glycerol broth as stock.

Maintenance of media, reagents and solutions. Preservation and maintenance of different materials including microbial strains, heat labile chemicals and reagents like antibiotic disks were carried out in a refrigerator (vest frost, Germany) at $4{ }^{\circ} \mathrm{C}$. Trypticase Soy agar (TSA; Oxoid, UK), Nutrient Agar (NA; Oxoid, UK) and Eosine Methylene Blue Agar (EMB; Oxoid, UK) plates were used for the routine maintenance of the microbial strains. Stocks of the pure bacterial cultures were stored at $-20{ }^{\circ} \mathrm{C}$ in glycerol broth.

Resuscitation of bacteria from stock. Samples from glycerol broth were revived in NB. For resuscitation one loopful medium from glycerol broth was inoculated into Nutrient Broth (NB) and incubated at $37{ }^{\circ} \mathrm{C}$ for $24 \mathrm{hrs}$ for visible growth in broth. When visible growth appeared one loopful culture from NB was streaked on to NA and incubated for $24 \mathrm{hrs}$ at $37^{\circ} \mathrm{C}$.

Presumptive identification on EMB agar. An isolated colony on NA was streaked on to EMB for presumptive identification of the isolates. EMB plates were incubated at $37{ }^{\circ} \mathrm{C}$ for $24 \mathrm{hrs}$. Pink colonies with greenish metallic sheen were picked for further identification.

Preparation of stock solution of antibiotic. Stock solutions of Ceftriaxone, Cefotaxime and Imipenem at concentrations of $0.1 \mu \mathrm{g} / \mathrm{mL}$ were prepared. For the preparation of antibiotic stock solutions, powdered forms of antibiotics were used. Ceftriaxone (Drug International Ltd., Bangladesh), Cefotaxime (Incepta Ltd., Bangladesh), and Imipenem (SK-F Ltd, Bangladesh) powders were dissolved in distilled water. From the stock solution desired working concentrations were obtained for further work.

Preparation of turbidity standards (McFarland). McFarland 0.5 turbidity standards were prepared as per the standard guidelines described by the Clinical and Laboratory Standards Institute (5). A volume of $0.5 \mathrm{~mL}$ of a $1.175 \%(\mathrm{w} / \mathrm{v})$ Barium chloride dehydrate $\left(\mathrm{BaCl}_{2} .2 \mathrm{H}_{2} \mathrm{O}\right)$ solution was added to $99.5 \mathrm{~mL}$ of $0.18 \mathrm{~mol} / \mathrm{L}(1 \% \mathrm{v} / \mathrm{v})$ Sulfuric acid with constant stirring to maintain the suspension. The turbidity standard was then aliquoted into $4 \mathrm{~mL}$ vials, identical to those used to prepare the inoculum suspension. McFarland standards were stored in the dark at room temperature $\left(22{ }^{\circ} \mathrm{C}\right.$ to $\left.25^{\circ} \mathrm{C}\right)$. Before use, the standards were shaken well, mixing the fine white precipitate of barium sulfate in the tube.

Preparation of plates with antibiotic solution. At first, $20 \mathrm{~mL}$ sterile Mueller Hinton Agar (MHA) was measured with a sterile measuring cylinder. Then required concentration of antibiotics was poured into sterile petri dishes with a micropipette. Next step was to pour the media into petri dishes. Then antibiotic solution was mixed with MHA by rotating the petri dishes on working surface.

Procedure of MIC. At first the bacteria were streaked from glycerol broth stocks on to NA and incubated at $37{ }^{\circ} \mathrm{C}$ for $24 \mathrm{hrs}$. Next day from NA the bacteria were streaked on to EMB agar and incubated at $37{ }^{\circ} \mathrm{C}$ for $24 \mathrm{hrs}$. Bacteria inoculated into MHB within sterile $4 \mathrm{ml}$ vials from these EMB plates were incubated at $37{ }^{\circ} \mathrm{C}$ for $4 \mathrm{hrs}$. The loop was burned every time before and after inoculating the culture. The turbidity of actively growing cultures in fresh MHB was adjusted to obtain the turbidity of McFarland 0.5 standard. From these turbidity of McFarland 0.5 standard culture, the bacteria were patched with sterile tips on to MHA containing different concentration of antibiotics with a numbered grid line attached on the bottom of each plate. The plates were then incubated at $37^{\circ} \mathrm{C}$ for $24 \mathrm{hrs}$. After $24 \mathrm{hrs}$ the plates were observed for the presence or absence of growth in presence of different concentration of antibiotics.

ESBL assay. ESBL was assayed according to the procedure outlined in the CLSI guidelines (5). Bacteria were streaked from glycerol broth stocks on to NA and incubated at $37{ }^{\circ} \mathrm{C}$ for $24 \mathrm{hrs}$. Next day the bacteria were streaked from NA on to EMB agar and incubated at $37{ }^{\circ} \mathrm{C}$ for $24 \mathrm{hrs}$. A single colony was inoculated into $2 \mathrm{ml}$ MHB within sterile vials from these EMB plates and incubated at $37{ }^{\circ} \mathrm{C}$ for 4 hrs. The loop was burned every time before and after inoculating the culture to maintain sterility. The turbidity of actively growing cultures in fresh MHB was adjusted to obtain the turbidity of McFarland 0.5 standard. From these bacteria were spread in the form of a lawn onto MHA plates containing disks of antibiotics of Ceftriaxone, Cefotaxime, Amoxycillin and amoxycillin-clavulanic acid. The disks were placed over the lawn of MHA with a sterile applicator. The applicator was burned each time before placing the disks on to the bacterial lawns for sterility. The amoxycillin-clavulanic acid disks were placed in the middle of the plates. After that the plates were incubated at $37{ }^{\circ} \mathrm{C}$ for $24 \mathrm{hrs}$. Next day the plates were observed to determine the effect of ESBL (Extended Spectrum $\beta$-Lactamase).

Stability assay. Bacteria were streaked from glycerol broth stock on to NA and incubated at $37{ }^{\circ} \mathrm{C}$ for $24 \mathrm{hrs}$. Next day from NA the bacteria were streaked on to EMB agar and incubated at $37{ }^{\circ} \mathrm{C}$ for $24 \mathrm{hrs}$. Bacteria inoculated into $2 \mathrm{ml}$ MHB within sterile vials from these EMB plates were incubated at $37{ }^{\circ} \mathrm{C}$ for $4 \mathrm{hrs}$. The loop was burned every time before and after inoculating the culture to maintain sterility. The turbidity of actively growing cultures in fresh MHB was adjusted to obtain the turbidity of McFarland 0.5 standard. From these cultures were diluted to 100 and 10 folds using serial dilution technique to obtain isolated colonies. From these plate cultures 20 well isolated colonies were randomly selected and simultaneously patched with sterile tips on one MHA medium without antibiotic and with ceftriaxone $(1024 \mu \mathrm{g} / \mathrm{mL})$ with a numbered grid line attached on the bottom of each plate. After 24 hour incubation at $30{ }^{\circ} \mathrm{C}$ the plates were observed for the presence of growth in presence and absence of antibiotics. The same procedure was repeated for 21 days.

\section{RESULTS AND DISCUSSION}

\section{Minimum Inhibitory Concentrations (MICs) of tested} $\boldsymbol{\beta}$-lactam antibiotics against test $\boldsymbol{E}$. coli. A total of 20 isolates were tested to determine the MIC values for three antibiotics, viz. Ceftriaxone, Cefotaxime and Imipenem. The MIC values are presented in Table 1.

TABLE 1. Minimum Inhibitory Concentration (MIC) of antibiotics tested in the present study

\begin{tabular}{ccc}
\hline $\begin{array}{c}\text { Antibiotic } \\
\text { tested }\end{array}$ & $\begin{array}{c}\text { MIC } \\
(\mu \mathrm{g} / \mathrm{ml})\end{array}$ & $\begin{array}{c}\text { No. of } \\
\text { isolates }\end{array}$ \\
\hline Ceftriaxone & 1 & 2 \\
& 2 & 1 \\
& 4 & 1 \\
& 32 & 1 \\
& 2048 & 1 \\
& 4096 & 4 \\
& $>4096$ & 10 \\
\hline Cefotaxime & 1 & 4 \\
& 64 & 1 \\
& 2048 & 6 \\
& 4096 & 7 \\
& $>4096$ & 2 \\
\hline Imipenem & 1 & 16 \\
& 8 & 1 \\
& 2048 & 3 \\
\hline
\end{tabular}


The CLSI guideline in 2012 states that organisms resistant to Ceftriaxone are usually also resistant against Cefotaxime and vice versa (5). The finding of the present study was in agreement with the CLSI guidelines. In addition, the MIC values of the two antibiotics, Ceftriaxone and Cefotaxime, were also similar. The guideline states a value of $\geq 4 \mu \mathrm{g} / \mathrm{mL}$ of each of these antibiotics for resistant members of Enterobaceriaceae. CLSI guidelines (5) state that a MIC of $2 \mu \mathrm{g} / \mathrm{mL}$ indicates 'Intermediate resistance' to the antibiotic and that $\geq 4 \mu \mathrm{g} / \mathrm{ml}$ is considered the MIC of resistant isolates. Thus, the test isolates in the study show intermediate resistance to Imipenem, which is still a suitable candidate for treatment. A similar conclusion was drawn in another study conducted in Bangladesh. The authors concluded that regarding antimicrobial susceptibility, Imipenem is the most effective antibiotic for the management of infection by ESBL producers. They proceeded to suggest that an evaluation of combined therapy is required in order to determine the efficacy of treating infections by ESBL producers. Several studies have demonstrated that a modifiable risk factor for the development of ESBL producing organisms is the use of third-generation cephalosporins.

Hence, formulary modification by decreasing the use of third-generation cephalosporins and increasing the use of imipenem, meropenem with amikacin or piperacillin-tazobactam should significantly decrease the isolation of ESBL-producing bacteria. The ESBLproducing organisms are increasing rapidly and becoming a major problem in the area of infectious diseases (1).

Prevalence of ESBL producers. In the present study we found $8 \mathrm{ESBL}$ producers $(40 \%, \mathrm{n}=20), 14 \beta$ lactamase producers $(70 \%, \mathrm{n}=20), 2$ isolates which exhibited ESBL activity against Cefotaxime and ceftriaxone $(25 \%, \mathrm{n}=8), 3$ isolates which exhibited ESBL activity against only Cefotaxime $(37.5 \%, \mathrm{n}=8)$ and 3 isolate which exhibited ESBL activity against only ceftriaxone $(37.5 \%, \mathrm{n}=8$ ) among 20 clinical isolates (Figures 1 and 2). The emergence of extendedspectrum $\beta$-lactamases (ESBLs) in gram-negative bacteria has increased in recent years, which has led to global concern regarding the management of bacterial infections $(6,7)$.

Extended spectrum beta-lactamase (ESBL) are plasmid mediated, TEM-1, TEM-2 and SHV-1 derived enzymes conferring broad resistance to penicillin, cephalosporin and monobactam but not to carbapenem (3). These enzymes are produced by Enbterobacteriaceae mainly by Escherichia coli, Klebsiella pneumoniae and oxytoca (8). The ESBL enzymes are capable of hydrolyzing broad spectrum cephalosporins and monobactams but inactive against Cephamycins and

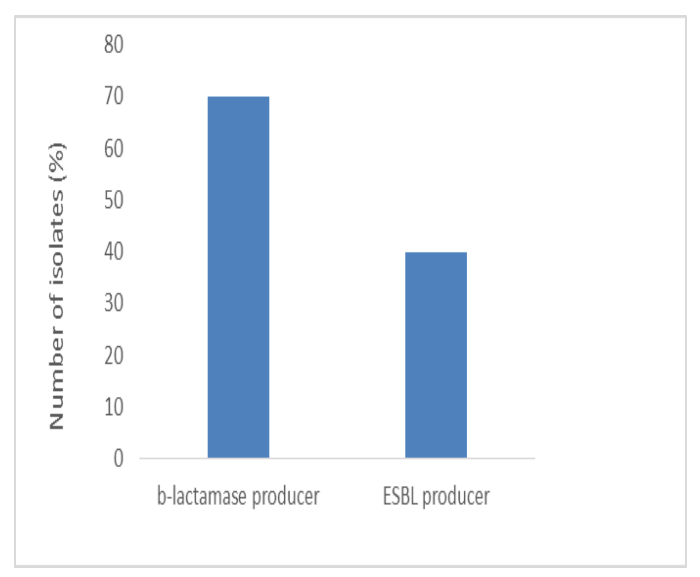

FIG 1. Proportion of $\beta$-lactamase and ESBL producers among test isolates

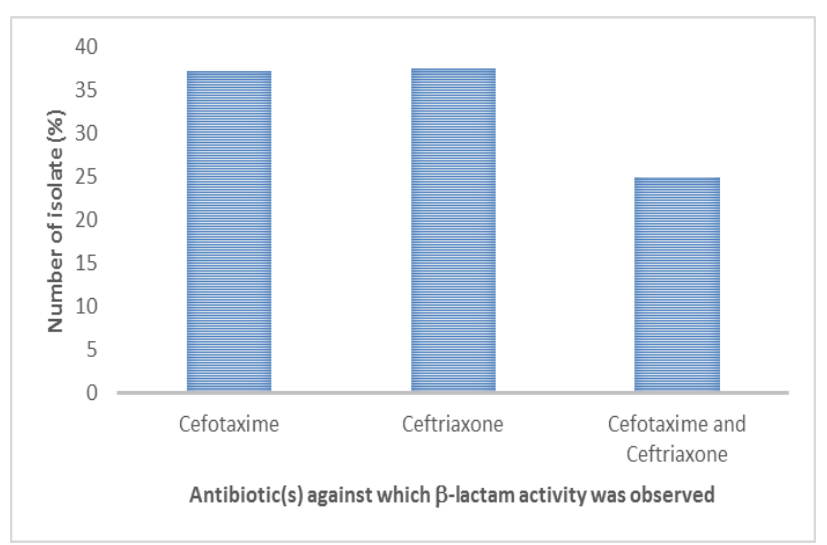

FIG 2. Antibiotics against which $\beta$-lactam activity was observed among test isolates

and Imipenem. In addition, ESBL producing organisms exhibit co-resistance to many other classes of antibiotics resulting in limitation of therapeutic option (1). Recent studies revealed that patients with infection such as septicaemia with ESBL producing organisms had significantly higher fatality rate than those with nonESBL isolates (3). Though the prevalence of ESBL producers varies with geographical areas and time, a relatively high prevalence rate of ESBL producers in the Asia-Pacific area were documented by several surveillance studies $(9,10)$. Previous studies in Bangladesh reported the prevalence of ESBL producers ranging from $23.2 \%$ to $80 \%(4,11,13)$. The discrepancy of the findings between the latter and the present study may be due to the varying prevalence of ESBL producers with time as well as from country to country, city to city and even hospital to hospital in one city. In spite of this, the finding of $40 \%$ ESBL producers in the present study is therefore in concordance with earlier reports. Currently, over 300 different ESBLs have been identified in gram negative bacilli (12). At present, blaCTX-M is considered as the most frequent type of ESBLs worldwide. Another 
Another growing family of ESBLs, oxacillin hydrolyzing (OXA) types have been reported, which are not usually inhibited by clavulanic acid except OXA-18 and OXA-45 (12, 14, 15). According to CDC (2012), ESBLs compromise the effects of penicillin, first-, second-, third-generation cephalosporins and monobactams but do not affect cephamycin or carbapenem. In the present study, none of the ESBL producers exhibited any activity against Imipenem, a carbapenem. This finding is similar to the report by CDC at 2012 on the lack of activity of ESBL producers on cephamycin or carbapenems.

Stability of antibiotic resistance. It was found that at $1024 \mu \mathrm{g} / \mathrm{mL}$ concentration of ceftriaxone, 10 isolates of clinical E.coli were resistant to Ceftriaxone for upto 21 days. These isolates were labelled as 1, 3, 4, 7, 8, 11, 12, 13, 14 and 15 which were selected randomly. E. coli DH5 $\boldsymbol{\alpha}$ was used as a negative control, as it was sensitive to Ceftriaxone.

\section{CONCLUSION}

The present study was conducted to investigate the occurrence of ESBL among clinical E. coli isolates. Based on the findings of the present study it can be concluded that test isolates of $E$. coli were resistant to $\beta$ lactam antibiotics viz. Ceftriaxone, Cefotaxime and mainly intermediately resistant to Imipenem. The MICs of Ceftriaxone and Cefotaxime were found to be between 2048 and $\geq 4096 \mu \mathrm{g} / \mathrm{mL}$ level, whereas that for Imipenem was $2048 \mu \mathrm{g} / \mathrm{ml}$. The prevalence of ESBL producers was $40 \%$, in agreement with other reports on ESBL prevalence by other workers. $\beta$-lactam resistance is stable for until 21 days of stability assay in laboratory medium. In Bangladesh, detection of ESBL producers is not routinely conducted because of deficiency of resources and facilities in most of the laboratories. Significant antimicrobial resistance among the ESBL producers suggests the urgent necessity for inclusion of detection of ESBL producers in routine laboratories. The finding of this study indicates an urgent surveillance of ESBL producers in larger number of clinical isolates in order to obtain an accurate prevalence of ESBL producers in Bangladesh. In addition, increasing reports of carbapenem resistant isolates provide further necessity for monitoring and controlling infections caused by ESBL producing bacteria.

\section{ACKNOWLEDGEMENTS}

The author would like to express thanks to the Department of Microbiology, University of Dhaka for laboratorv facilities.

\section{REFERENCES}

1. Alipourfard I, Nili N. 2010. Antibiogram of Extended Spectrum Betalactamase (ESBL) producing Escherichia coli and Klebsiella pneumoniae isolated from Hospital Samples. Bangladesh. J. Med Microbiol. 4 (1): 32-36.

2. Poole K. 2007. Efflux pumps as antimicrobial resistance mechanisms. Ann Med. 39 (3): 162-76.

3. Mehrgan H, Rahbar M. 2008. Prevalence of extended-spectrum betalactamase producing Escherichia coli in a tertiary care hospital in Tehran, Iran. Int. J. Antimicrob. Agents. 31: 147-151.

4. Farzana R, Shamsuzzaman SM, Mamun KZ, Shears P. 2013. Antimicrobial susceptibility pattern of Extended Spectrum $\beta$-Lactamase producing Gram negative bacteria isolated from wound and urine in a tertiary care hospital, Dhaka city, Bangladesh. Southeast Asian J. Trop. Med. Public Health. 44 (1): 96-103.

5. CLSI. 2012. Performance Standards for Antimicrobial Disk Susceptibility Tests; Approved Standard-Eleventh Edition. CLSI document M02-A11. Clinical and Laboratory Standards Institute, Wayne, PA.

6. Paterson DL. 2006. Resistance in gram-negative bacteria: Enterobacteriaceae. Am J Med. 119: S20-S28.

7. Bourjilat F, Bouchrif B, Dersi N, Gros Claude JD, Amarouch H, Timinouni M. 2011. Emergence of extended-spectrum beta-lactamasesproducing Escherichia coli in community-acquired urinary infections in Casablanca, Morocco. J. Infect. Dev. Ctries. 5: 850-5.

8. Philippon A, Labia R, Jacoby G. 1989. Extended-spectrum lactamases. Antimicrob Agents Chemother. 33: 1131-6.

9. Hirakata, Y, Matsuda J, Miyazaki Y, Kamihira S, Kawakami S, Miyazawa Y, et al. 2005. Regional variation in the prevalence of extendedspectrum beta-lactamase- producing clinical isolates in the Asia Pacific region (SENTRY 1998-2002). Diagn. Microboil. Infect. Dis. 52: 323-9.

10. Bell JM, Turnidge JD, Gales AC, Pfaller MA, Jones RN. 2002. Prevalence of extended spectrum beta lactamase (ESBL)-producing clinical isolates in the Asia-Pacific region and South Africa: regional results from SENTRY Antimicrobial Surveillance Program (1998-99). Diagn. Microbiol. Infect. Dis. 42: 193-8.

11. Islam, S. 2008. Detection of extended-spectrum beta-lactamases producing organisms with their phenotypic confirmation by $\mathrm{E}$ test and susceptibility to quinolone and fluoroquinolones. M Phil thesis, University of Dhaka, Dhaka, Bangladesh.

12. Rawat, D, Nair D. 2010. Extended-spectrum b-lactamases in gram-negative bacteria. J Glob Infect Dis. 2: 263-74.

13. Biswas S. 2009. Comparison of three dimensional test and double disc synergy test for detection of extended-spectrum $\beta$-lactamase-producing Gram negative bacteria. M Phil thesis, Bangabandhu Sheikh Mujib Medical University, Dhaka.

14. Philippon LN, Naas T, Bouthors AT, Barakett V, Nordmann P. 1997. OXA-18, a class D clavulanic acid-inhibited extended-spectrum betalactamase from Pseudomonas aeruginosa. Antimicrob Agents Chemother. 41: 2188-95.

15. Toleman MA, Rolston K, Jones RN, Walsh TR. 1988. Molecular and biolchemical characterization of OXA-45, an extended-spectrum class $2 \mathrm{~d}$ ' beta-lactamase in Pseudomonas aeruginosa. Antimicrob Agents Chemother conferring transferable resistance to newer-lactam agents in Enterobacteriaceae: hospital prevalence and susceptibility patterns. Rev Infect Dis. 10: 867-78.

16. Cisneros JM, Rodríguez-Baño J, Fernández-Cuenca F, Ribera A, Vila J, Pascual A, et al. 2005. Risk-factors for the acquisition of imipenemresistant Acinetobacter baumannii in Spain: a nationwide study. Clin. Microbiol. Infect. 11: 874-9.

17. Meyer E, Jonas D, Schwab F, Rueden H, Gastmeier P, Daschner FD. 2003. Design of a surveillance system of antibiotic use and bacterial resistance in German intensive care units (SARI). Infection. 31: 208-15. 